\title{
Postoperative adjuvant chemotherapy combined with intracavitary brachytherapy in early-stage cervical cancer patients with intermediate risk factors
}

\author{
This article was published in the following Dove Press journal: \\ OncoTargets and Therapy \\ I December 2016 \\ Number of times this article has been viewed
}

$\mathrm{Hao} \mathrm{Yu}^{\prime}$

Linlin Zhang ${ }^{2}$

Xuelian Du'

Xiugui Sheng'

'Department of Gynecologic Oncology, Shandong Cancer Hospital and Institute Affiliated to Shandong University, ${ }^{2}$ Department of Obstetrics and Gynecology, Jinan Fourth People's Hospital, Jinan, People's Republic of China
Correspondence: Xiugui Sheng Department of Gynecologic Oncology, Shandong Cancer Hospital and Institute Affiliated to Shandong University, 440 Jiyan Road, 250 I I7, Jinan,

People's Republic of China Email jnsxg@hotmail.com
Objective: To investigate the impact of postoperative adjuvant therapy on survival of patients with intermediate risk early-stage cervical squamous cell carcinoma.

Methods: A total of 133 consecutive patients with intermediate risk early-stage cervical squamous cell carcinoma treated at Shandong Cancer Hospital and Institute from February 2010 to March 2014 were enrolled in our study. All patients received adjuvant therapy and were subdivided into three groups: pelvic radiotherapy ( $R T$; $N=42$ ), adjuvant chemotherapy + intracavitary radiotherapy (CT+ICRT; $\mathrm{N}=47$ ), or concurrent chemoradiation (CCRT; $\mathrm{N}=44$ ). Disease-free survival (DFS) and therapeutic complications were evaluated.

Results: There were no significant differences in DFS for patients treated with RT, CT+ICRT, and CCRT $(P>0.05)$ with 3-year rates of $94.0 \%, 93.4 \%$, and $97.6 \%$, respectively. Frequencies of grade III-IV acute toxicities were higher in patients treated with CCRT (34.1\%) than those treated with RT $(9.5 \%)$ or CT+ICRT $(16.7 \% ; P<0.05)$, with no significant differences observed between RT and CT+ICRT groups $(P>0.05)$. Grade I-II late toxicities were higher in CCRT (25\%), followed by RT $(19.0 \%)$, and finally, the CT+ICRT group $(4.3 \% ; P<0.05)$; with no significant differences observed between CCRT and RT groups $(P>0.05)$.

Conclusion: Treatment with CT+ICRT or RT resulted in the equivalent of 3-year DFS compared to CCRT, but fewer therapeutic complications were observed with CT for patients with intermediate risk early-stage cervical squamous cell carcinoma.

Keywords: cervical squamous cell carcinoma, radiotherapy, chemotherapy, concurrent chemoradiotherapy

\section{Introduction}

Radical hysterectomy and pelvic lymphadenectomy are typically the primary treatment strategies for early-stage cervical cancer and offer similar 5-year survival rates as radical radiation (RT). Patients with adverse prognostic factors as identified via postoperative pathological examinations are often treated with RT, chemotherapy $(\mathrm{CT})$, or concurrent chemoradiation (CCRT). ${ }^{1}$ In 2000, Peters et $\mathrm{al}^{2}$ reported a significant survival benefit associated with CCRT rather than RT alone in patients with high risk factors, such as parametrial invasion, lymph node metastasis, and positive surgical margin. Until now, CCRT has been considered a primary postoperative therapy for high-risk cervical cancer. ${ }^{3}$ However, the treatment regimen for patients with intermediate risk factors, including deep stromal invasion (DSI), lymph vascular space involvement (LVSI), low differentiation, and tumor diameters $\geq 4 \mathrm{~cm}$ remains unclear. 
Considering the acute or late complications associated with RT, we evaluated the efficacy and potential complications of postoperative adjuvant CT combined with intracavitary brachytherapy in early-stage cervical cancer patients with 1-2 intermediate risk factors.

\section{Materials and methods \\ Patients}

A total of 133 consecutive patients treated at the Shandong Cancer Hospital and Institute between February 2010 and March 2014 were enrolled in the current study. Patients provided written informed consent and the study was approved by the Human Ethics Committee of Shandong Cancer Hospital and Institute. Inclusion criteria were as follows: the International Federation of Gynecology and Obstetrics stage Ia-IIa cervical squamous carcinoma; no treatment prior to surgery; history of radical hysterectomy and pelvic lymphadenectomy; and 1-2 intermediate risk factors including DSI, LVSI, tumor diameters $\geq 4 \mathrm{~cm}$, and low differentiation. All patients received adjuvant therapy and were subdivided into three groups: pelvic RT (RT group; $\mathrm{N}=42$ ), adjuvant $\mathrm{CT}+$ intracavitary radiotherapy (ICRT) (CT+ICRT group; $\mathrm{N}=47$ ), and CCRT (CCRT group; $\mathrm{N}=44)$ (Table 1).

Table I Clinical and pathologic characteristics of the patients with intermediate risk factors according to different adjuvant therapies

\begin{tabular}{|c|c|c|c|c|}
\hline Patient characteristics & $\begin{array}{l}R T \\
(n=42)\end{array}$ & $\begin{array}{l}\text { CT+ICRT } \\
(n=47)\end{array}$ & $\begin{array}{l}\text { CCRT } \\
(n=44)\end{array}$ & $P$-value \\
\hline Age & & & & $>0.05$ \\
\hline Premenopausal & 28 & 29 & 29 & \\
\hline Postmenopausal & 14 & 18 & 15 & \\
\hline Anemia & & & & $>0.05$ \\
\hline Yes & 12 & 11 & 10 & \\
\hline No & 30 & 36 & 34 & \\
\hline FIGO stage & & & & $>0.05$ \\
\hline la & 4 & 5 & 3 & \\
\hline $\mathrm{lb}$ & 20 & 26 & 24 & \\
\hline lla & 18 & 16 & 17 & \\
\hline $\begin{array}{l}\text { Intermediate risk factors } \\
\text { (one) }\end{array}$ & & & & $>0.05$ \\
\hline$>4 \mathrm{~cm}$ & 8 & 9 & 16 & \\
\hline LVSI & 12 & 10 & 12 & \\
\hline DSI & 23 & 24 & 20 & \\
\hline LD & 18 & 16 & 22 & \\
\hline $\begin{array}{l}\text { Intermediate risk factors } \\
\text { (two) }\end{array}$ & 15 & 17 & 19 & $>0.05$ \\
\hline
\end{tabular}

Abbreviations: RT, radiotherapy; CT, chemotherapy; ICRT, intracavitary radiotherapy; CCRT, concurrent chemoradiation; FIGO, International Federation of Gynecology and Obstetrics; LVSI, lymph vascular space involvement; DSI, deep stromal invasion; LD, low differentiation.

\section{Pelvic RT}

RT consisted of pelvic external irradiation and ICRT. Pelvic external irradiation was administered 3-4 weeks after the operation using a 15 MV X-ray from a linear accelerator. The superior margin of the field was extended to the upper border of the fifth lumbar vertebra. The inferior margin was the inferior border of the obturator foramen, and laterally, the field extended $2 \mathrm{~cm}$ beyond the lateral margin of the bony pelvic wall. A total dose of 45.0-50.0 Gy was administered over 5-6 weeks with 1.8-2 Gy per fraction, five fractions per week. After application of 25-30 Gy, the center of the pelvic field was blocked 4-6 cm in width, and ICRT was delivered using Ir192 with 2.5-3.0 Gy per fraction, two fractions per week for a total dose of 20.0-25.0 Gy at $5 \mathrm{~mm}$ below the vaginal mucosa.

\section{Adjuvant CT+ICRT}

The CT regimen consisted of paclitaxel $\left(135 \mathrm{mg} / \mathrm{m}^{2}\right.$, intravenous) for 24 hours on day 1 , followed by carboplatin (area under the curve 5-6) on day 2. Patients received 4-6 courses at 3-week intervals beginning 2 weeks after surgery. ICRT using Ir192 with 2.5-3.0 Gy per fraction, two fractions per week for a total dose of 20.0-25.0 Gy at $5 \mathrm{~mm}$ below the vaginal mucosa was administered.

\section{CCRT}

The treatment modality of RT and ICRT was consistent with the descriptions stated above. Carboplatin $\left(150 \mathrm{mg} / \mathrm{m}^{2}\right)$ and paclitaxel $\left(60 \mathrm{mg} / \mathrm{m}^{2}\right)$ were administered (intravenously) on a weekly basis during the course of RT for 5-6 weeks.

\section{Toxicity}

Chemotherapeutic toxicity was evaluated according to the World Health Organization's guidelines. Acute and late normal tissue effects were graded according to the Radiation Therapy Oncology Group radiation morbidity scoring criteria.

\section{Follow-up}

After treatment completion, patients were followed-up in an outpatient clinic every month in the first year, every 3 months in the second year, every 3-6 months in the third year, and every 6 months in the fourth and fifth years. The follow-up schedule consisted of physical and pelvic examinations, Pap smears, vaginal ultrasounds, and chest X-rays. Computed tomography and magnetic resonance imaging were performed if necessary suspect. Recurrence was defined as the appearance of clinical, radiologic, or histologic evidence of disease after study entry. 


\section{Statistical methods}

Patient survival was calculated using the Kaplan-Meier method. The significance of the survival difference was evaluated using the log-rank test. A $\chi^{2}$ test was used to analyze correlations among variables. Significance was set at $P<0.05$. Statistical analyses were performed with the SPSS 13.0 software package (SPSS Inc., Chicago, IL, USA).

\section{Results}

The median follow-up period was 30 months (range, 6-56 months). Six patients were lost to follow-up (three subjects in the RT group, two subjects in the CT+ICRT group, and one subject in the CCRT group). As shown in Figure 1, the 3-year disease-free survival (DFS) rates in the RT, CT+ICRT, and CCRT groups were 94.0\% (95\% confidence interval [CI], 50.689-55.637), 93.4\% (95\% CI, 47.492-54.311), and 97.6\% (95\% CI, 48.921-52.982), respectively (Figure 1), with no statistical differences among the three groups $(P>0.05)$. In the RT group, two patients had pelvic tumor recurrence at the 20th and 28th month (recurrence rate $4.7 \%$ ). In the $\mathrm{CT}+\mathrm{ICRT}$ group, three patients had pelvic tumor recurrence, with two subjects at the 2 nd and one subject at the 17 th month (recurrence rate 6.3\%). In the CCRT group, one patient had pelvic tumor recurrence in the 9th month (recurrence rate $2.3 \%$ ). The site of recurrence was the pelvis including the vaginal stump, pelvic wall, or pelvic lymph nodes. No distant recurrence was observed and no patients died due to cancer (Table 2).

Patients treated with CCRT reported more adverse events. Acute toxicities included myelosuppression, and gastrointestinal and hepatic toxicity. Myelosuppression

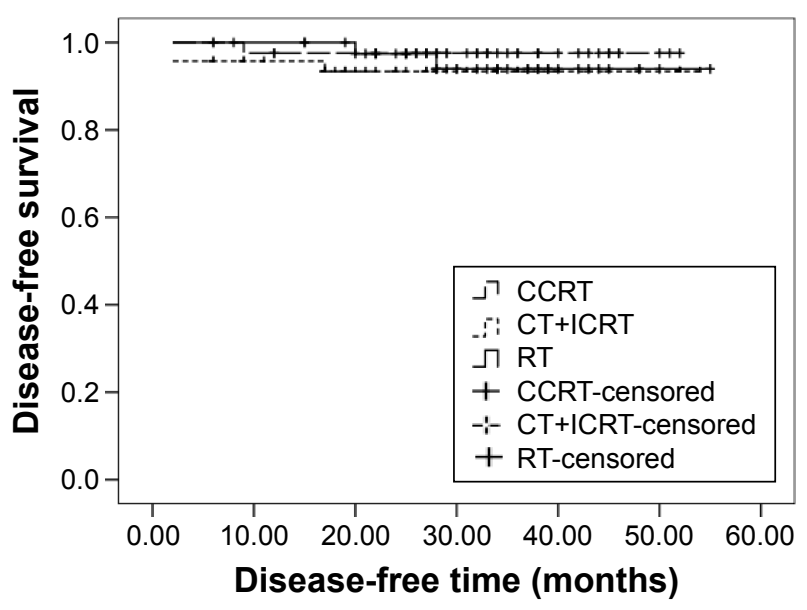

Figure I Three-year disease-free survival of patients with intermediate risk factors according to different adjuvant therapies.

Abbreviations: $\mathrm{RT}$, radiotherapy; $\mathrm{CT}$, chemotherapy; ICRT, intracavitary radiotherapy; CCRT, concurrent chemoradiation.
Table 2 Relapse rates and sites of failure

\begin{tabular}{lll}
\hline Groups & Distant recurrence & Pelvic recurrence \\
\hline RT $(n=42)$ & 0 & $2(4.7 \%)$ \\
CT + ICRT $(n=47)$ & 0 & $3(6.3 \%)$ \\
CCRT $(n=44)$ & 0 & $1(2.3 \%)$ \\
\hline
\end{tabular}

Abbreviations: RT, radiotherapy; CT, chemotherapy; ICRT, intracavitary radiotherapy; CCRT, concurrent chemoradiation.

mainly consisted of leukopenia and low platelet counts. The incidences of grade III to IV myelosuppression were $2.4 \%$, $8.5 \%$, and $15.9 \%$, in the RT, CT+ICRT, and CCRT groups, respectively. Gastrointestinal toxicity mainly included nausea, vomiting, and diarrhea. The incidences of grade III to IV gastrointestinal toxicity were $7.1 \%, 4.3 \%$, and $11.4 \%$ in the RT, CT+ICRT, and CCRT groups, respectively. Hepatic toxicity mainly included transaminase abnormalities. The incidences of grade III to IV hepatic toxicity were $0 \%, 2.1 \%$, and $6.8 \%$ in the RT, CT+ICRT, and CCRT groups, respectively. The frequencies of grade III to IV acute toxicities in the CCRT group (34.1\%) were higher compared with the RT group (9.5\%) or the CT+ICRT group $(16.7 \%)(P<0.05)$. No statistical difference was observed between the RT and the $\mathrm{CT}+\mathrm{ICRT}$ groups $(P>0.05)$. The incidences of grade I to II chronic proctitis were $11.9 \%, 4.3 \%$, and $13.6 \%$; and $7.1 \%$, $0 \%$, and $11.4 \%$ for chronic cystitis in the RT, CT+ICRT, and CCRT groups, respectively. Grade I to II late toxicity was significantly more frequent in the CCRT $(25 \%)$ and the RT (19.0\%) groups compared with the CT+ICRT group $(4.3 \%)(P>0.05)$, but this was not statistically significant $(P>0.05)$ (Table 3).

\section{Discussion}

A prospective study conducted by the Gynecological Oncology Group (GOG) analyzing the relationship of

Table 3 Complications of patients with intermediate risk factors according to different adjuvant therapies

\begin{tabular}{|c|c|c|c|c|}
\hline Complication & $\begin{array}{l}\text { RT } \\
(n=42)\end{array}$ & $\begin{array}{l}C T+I C R T \\
(n=47)\end{array}$ & $\begin{array}{l}\text { CCRT } \\
(n=44)\end{array}$ & $P$-value \\
\hline Acute toxicities & $4(9.5 \%)$ & 7 (I6.7\%) & $15(34.1 \%)$ & $<0.05$ \\
\hline \multicolumn{5}{|l|}{ (Grade III-IV) } \\
\hline Myelosuppression & I (2.4\%) & $4(8.5 \%)$ & 7 (I5.9\%) & \\
\hline Gastrointestinal & $3(7.1 \%)$ & $2(4.3 \%)$ & 5 (II.4\%) & \\
\hline $\begin{array}{l}\text { Hepatotoxicity and } \\
\text { nephrotoxicity }\end{array}$ & $0(0 \%)$ & I (2.1\%) & $3(6.8 \%)$ & \\
\hline Late toxicities (grade I-II) & $8(19.0 \%)$ & $2(4.3 \%)$ & II (25\%) & $<0.05$ \\
\hline $\begin{array}{l}\text { Chronic radioactive } \\
\text { proctitis }\end{array}$ & 5 (11.9\%) & $2(4.3 \%)$ & $6(13.6 \%)$ & \\
\hline $\begin{array}{l}\text { Chronic radioactive } \\
\text { cystitis }\end{array}$ & $3(7.1 \%)$ & $0(0 \%)$ & 5 (II.4\%) & \\
\hline
\end{tabular}

Abbreviations: $\mathrm{RT}$, radiotherapy; $\mathrm{CT}$, chemotherapy; ICRT, intracavitary radiotherapy; CCRT, concurrent chemoradiation. 
pathological factors with disease free interval suggested that tumor size, LVSI, and the depth of tumor invasion were all independent prognostic factors for cervical cancer. By analyzing the relative risk (RR) of these three independent factors, patients can be classified in the low $(\mathrm{RR}<40)$, intermediate ( RR 40-120), or high $(>120)$ risk of recurrence categories. ${ }^{4}$ Adjuvant therapy should therefore be considered for intermediate and high-risk patients as they have a $20 \%$ and $41 \%$ risk of recurrence, respectively. Based on the GOG prognostic scoring system, Pieterse et $\mathrm{al}^{5}$ investigated the role of postoperative RT in early-stage cervical carcinoma with at least two of the aforementioned three risk factors. Those authors reported that postoperative RT reduced recurrence from $41 \%$ to $12 \%$, and the 5-year cancer-specific survival increased from $57 \%$ to $86 \%$, while the 5 -year DFS increased from $43 \%$ to $85 \%$. Based on those findings, the study concluded that cervical cancer patients with at least two risk factors significantly benefited from postoperative RT.

During the 1990s, the GOG conducted another prospective randomized trial protocol to investigate the role of RT for Ib stage cervical carcinoma with $\geq 2$ low-risk factors. Data from the initial report suggested that pelvic irradiation might reduce local recurrence, ${ }^{6}$ but final results published in 2006 indicated that RT could improve the recurrence rate and DFS of patients with at least two low-risk factors, while no significant benefit was observed in overall survival. ${ }^{7}$

Considering the serious complications and little overall survival benefit of RT for patients with cervical cancer, several studies have been performed to investigate the effectiveness of $\mathrm{CT}$ alone as postoperative adjuvant therapy. ${ }^{8-10}$ Takeshima et $\mathrm{al}^{11}$ investigated the value of $\mathrm{CT}$ as adjuvant therapy in 30 patients with DSI, and found that CT alone achieved 5-year DFS of $93.3 \%$ and the incidence of locoregional recurrence was $3.3 \%$. Even for patients with high-risk factors including parametrial invasion, lymph node metastasis, and positive surgical margin, CT alone yielded a 5-year DFS of $63 \% .{ }^{12}$ Hosaka et $\mathrm{al}^{13}$ conducted a retrospective study to compare the role of CT with RT in patients without multiple lymph node metastases, but with other recurrent risk factors, including DSI, LVSI, parametrial invasion, lymph node metastasis, and bulky tumors $(1-4 \mathrm{~cm})$. The 3 -year DFS with CT was $96.3 \%$, which was equivalent to $82.6 \%$ of RT, but with fewer postoperative complications such as bowel obstruction and urinary disturbance.

As postoperative adjuvant therapy of cervical cancer, RT was used to reduce the risk of locoregional recurrence and CT to suppress distant metastasis. Therefore, choosing CT, instead of RT, as a postoperative adjuvant therapy raises the possibility that this strategy may increase the risk of local recurrence. Thus, herein, intracavitary brachytherapy, combined with adjuvant $\mathrm{CT}$, was used to reduce locoregional recurrence, especially vaginal recurrence. We observed that the 3-year DFS in the CT+ICRT group was 93.4\%, which was equivalent to the CCRT and the RT groups. Pelvic relapse was observed in six patients and no distant metastasis was found (local recurrence was $4.7 \%, 6.3 \%$, and $2.3 \%$ in the RT, CT+ICRT, and CCRT groups, respectively).

Currently, radical hysterectomy is a primary therapy for early-stage cervical cancer. ${ }^{14}$ Additional adjuvant treatment, such as RT, CT, or CCRT, is considered for a patient based on their risk factors. Combining RT with surgery significantly increased the risk of complications such as leg edema, bowel obstruction, urinary tract dysfunction, or other late toxicities. ${ }^{15}$ Postsurgical intra-abdominal adhesion was responsible for the majority of those complications. ${ }^{16}$

Acknowledging physical differences between Western and Asian patients, radical hysterectomy may be sufficient for disease control of the pelvis. Regarding quality of life and favorable prognoses for those with intermediate risk factors, we investigated the role of CT as postoperative adjuvant therapy for cervical cancer patients with intermediate risk factors. Our results indicated that CT had an equivalent of 3-year PFS compared to RT and CCRT, but with fewer complications. The frequencies of acute grade III-IV toxicities were significantly higher in patients treated with CCRT $(30.5 \%)$ than in those treated with RT $(7.9 \%)$ or CT (12.9\%) alone. No significant difference was observed between RT and CT. Regarding frequencies of grade I-II late toxicities, both CCRT (25\%) and RT (18.4\%) were higher than CT (3.2\%), but no difference was observed between CCRT and RT.

In summary, our study examined the role of postoperative adjuvant CT combined with intracavitary brachytherapy for treatment of early-stage cervical cancer patients with intermediate risk factors. These treatment strategies achieved the equivalent of 3-year PFS compared with CCRT and RT, but with lower acute and late-grade toxicities. Therefore, CT combined with intracavitary brachytherapy may be worth reconsidering as an alternative postoperative therapy for patients with cervical cancer. Due to the nonrandomized design of the study, a trial with an expanded sample size and longer follow-up period is required to confirm our initial observations.

\section{Acknowledgments}

This work was supported by Research Foundation of Shandong Cancer Hospital and Institute. 


\section{Disclosure}

The authors declare that they have no conflict of interest.

\section{References}

1. Dávila Fajardo R, van Os R, Buist MR, et al. Post-operative radiotherapy in patients with early stage cervical cancer. Gynecol Oncol. 2014; 134(1):52-59.

2. Peters WA 3rd, Liu PY, Barrett RJ 2nd, et al. Concurrent chemotherapy and pelvic radiation therapy compared with pelvic radiation therapy alone as adjuvant therapy after radical surgery in high-risk early-stage cancer of the cervix. J Clin Oncol. 2000;18(8):1606-1613.

3. Mabuchi S, Morishige K, Isohashi F, et al. Postoperative concurrent nedaplatin-based chemoradiotherapy improves survival in early-stage cervical cancer patients with adverse risk factors. Gynecol Oncol. 2009; 115(3):482-487.

4. Delgado G, Bundy B, Zaino R, et al. Prospective surgical-pathological study of disease-free interval in patients with stage IB squamous cell carcinoma of the cervix: a Gynecologic Oncology Group study. Gynecol Oncol. 1990;38(3):352-357.

5. Pieterse QD, Trimbos JB, Dijkman A, et al. Postoperative radiation therapy improves prognosis in patients with adverse risk factors in localized, early-stage cervical cancer: a retrospective comparative study. Int J Gynecol Cancer. 2006;16(3):1112-1118.

6. Sedlis A, Bundy BN, Rotman MZ, et al. A randomized trial of pelvic radiation therapy versus no further therapy in selected patients with stage IB carcinoma of the cervix after radical hysterectomy and pelvic lymphadenectomy: A Gynecologic Oncology Group Study. Gynecol Oncol. 1999;73(2):177-183.

7. Rotman M, Sedlis A, Piedmonte MR, et al. A phase III randomized trial of postoperative pelvic irradiation in stage IB cervical carcinoma with poor prognostic features. Int J Radiat Oncol Biol Phys. 2006;65(1): 169-176.
8. Jung PS, Kim DY, Lee SW, et al. Clinical Role of Adjuvant Chemotherapy after Radical Hysterectomy for FIGO Stage IB-IIA Cervical Cancer: Comparison with Adjuvant RT/CCRT Using Inverse-Probability-ofTreatment Weighting. PLoS One. 2015;15;10(7):e0132298.

9. Shimada M, Nishimura R, Hatae M, et al. Comparison of adjuvant chemotherapy and radiotherapy in patients with cervical adenocarcinoma of the uterus after radical hysterectomy: SGSG/TGCU Intergroup surveillance. Eur J Gynaecol Oncol. 2013;34(5):425-428.

10. Lee TY, Jeung YJ, Lee CJ, et al. Promising treatment results of adjuvant chemotherapy following radical hysterectomy for intermediate risk stage 1B cervical cancer. Obstet Gynecol Sci. 2013;56(1):15-21.

11. Takeshima N, Umayahara K, Fujiwara K, et al. Treatment results of adjuvant chemotherapy after radical hysterectomy for intermediate- and high-risk stage IB-IIA cervical cancer. Gynecol Oncol. 2006;103(2): 618-622.

12. Mossa B, Mossa S, Marziani R. Adjuvant chemotherapy versus radiation therapy after radical surgery in high-risk positive node stage IB/IIA cervical cancer. Eur J Gynaecol Oncol. 2010;31(5):545-550.

13. Hosaka M, Watari H, Takeda M, et al. Treatment of cervical cancer with adjuvant chemotherapy versus adjuvant radiotherapy after radical hysterectomy and systematic lymphadenectomy. JObstet Gynaecol Res. 2008;34(4):552-556.

14. Alleyne-Mike K, van Wijk L, Hunter A. A retrospective review of patients with stage IB2 cervical cancer treated with radical radiation versus radical surgery as a primary modality. Int $J$ Gynecol Cancer. 2013;23(7):1287-1294.

15. Katepratoom C, Manchana T, Amornwichet N. Lower urinary tract dysfunction and quality of life in cervical cancer survivors after concurrent chemoradiation versus radical hysterectomy. Int Urogynecol J. 2014;25(1):91-96.

16. Van Goor H. Consequences and complications of peritoneal adhesions. Colorectal Dis. 2007;9 Supp1 2:25-34.
OncoTargets and Therapy

\section{Publish your work in this journal}

OncoTargets and Therapy is an international, peer-reviewed, open access journal focusing on the pathological basis of all cancers, potential targets for therapy and treatment protocols employed to improve the management of cancer patients. The journal also focuses on the impact of management programs and new therapeutic agents and protocols on

\section{Dovepress}

patient perspectives such as quality of life, adherence and satisfaction. The manuscript management system is completely online and includes a very quick and fair peer-review system, which is all easy to use. Visit http://www.dovepress.com/testimonials.php to read real quotes from published authors. 